\title{
Lattice Boltzmann simulation of water transport through Gas Diffusion Layers with homogeneous and heterogeneous PTFE distributions
}

\author{
Patrick Sarkezi-Selsky ${ }^{1 a}$, Henrike Schmies ${ }^{1 \mathrm{~b}}$, Arnulf Latz ${ }^{1 \mathrm{a}, 2}$, Thomas Jahnke ${ }^{1 \mathrm{a}}$ \\ (1) German Aerospace Center (DLR), Institute of Engineering Thermodynamics \\ aPfaffenwaldring 38-40, 70569 Stuttgart, Germany \\ ${ }^{b}$ Carl-von-Ossietzky-Straße 15, 26129 Oldenburg, Germany \\ (2) Helmholtz Institute Ulm for Electrochemical Energy Storage (HIU), \\ Albert-Einstein-Allee 11, 89081 Ulm, Germany \\ Tel.: $+497116862-498$ \\ Fax: $+497116862-747$ \\ Patrick.Sarkezi-Selsky@dlr.de
}

\begin{abstract}
In recent years Polymer Electrolyte Fuel Cells have gained increasing attention in the e-mobility sector being a compelling powertrain solution and alternative to batterydriven electrical vehicles. Short refueling periods, long range capacity and reliability at high loads amongst others render the fuel cell technology advantageous, especially for heavy duty applications such as public transport and commercial vehicles [1]. These use cases however come along with challenging demands for stable operation due to their dynamic load profiles. In fuel cell operation such requirements can only be fulfilled when reactant gases are steadily provided while at the same time product water is efficiently removed. This multiphase counterflow is typically realized using carbon-based gas diffusion layers (GDLs) coated with a hydrophobic additive easing water removal. However, multiphase flow in GDLs is very complex and underlying transport phenomena not yet sufficiently understood. In addition, material design and experimental testing is expensive and long-lasting. $A$ simulation approach offers therefore a large potential to deepen understanding of occurring transport phenomena while promoting material development.
\end{abstract}

In our work we simulate the flow of liquid water through porous gas diffusion media using a 3D color-gradient Lattice Boltzmann model [2]. The simulations are carried out on complex geometries as derived by binarization of $\mu \mathrm{CT}$ images for a real microstructure of a Freudenberg GDL. To investigate the influence of mixed wettability on multiphase flows a hydrophobic additive (PTFE) is furthermore included using an in-house distribution algorithm. With this routine different homogeneous and heterogeneous spatial distributions of PTFE are realized within the GDL. Imposing variable capillary pressures we then derive material dependent effective transport parameters such as capillary pressure-saturation curves for different additive contents and distributions. By simulating the consecutive intrusion and drainage of water we recover a characteristic hysteresis behavior for the liquid saturation depending on the PTFE distribution. These investigations contribute on the one hand to a better comprehension of transport mechanisms within GDLs. On the other hand 
can these effective transport parameters be used in cell-level simulation suites offering a more realistic description for multiphase flow in porous GDL material as compared to commonly used relations such as the standard Leverett approach [3].

The research leading to these results has received funding from the European Union's Horizon 2020 research and innovation program within the project ID Fast: Investigations on degradation mechanisms and Definition of protocols for PEM Fuel cells Accelerated Stress Testing, under grant agreement $n^{\circ} 779565$.

\section{Literature}

[1] Cullen, D.A., Neyerlin, K.C., Ahluwalia, R.K. et al., "New roads and challenges for fuel cells in heavy-duty transportation", Nat Energy, (2021), doi: 10.1038/s41560021-00775-z

[2] Rothman, D.H., Keller, J.M., "Immiscible cellular-automaton fluids", J. Stat. Phys. 52, 1119-1127 (1988), doi: 10.1007/BF01019743

[3] Leverett, M.C., "Capillary Behavior in Porous Solids", Trans. 142, 152-169 (1941), doi: 10.2118/941152-G

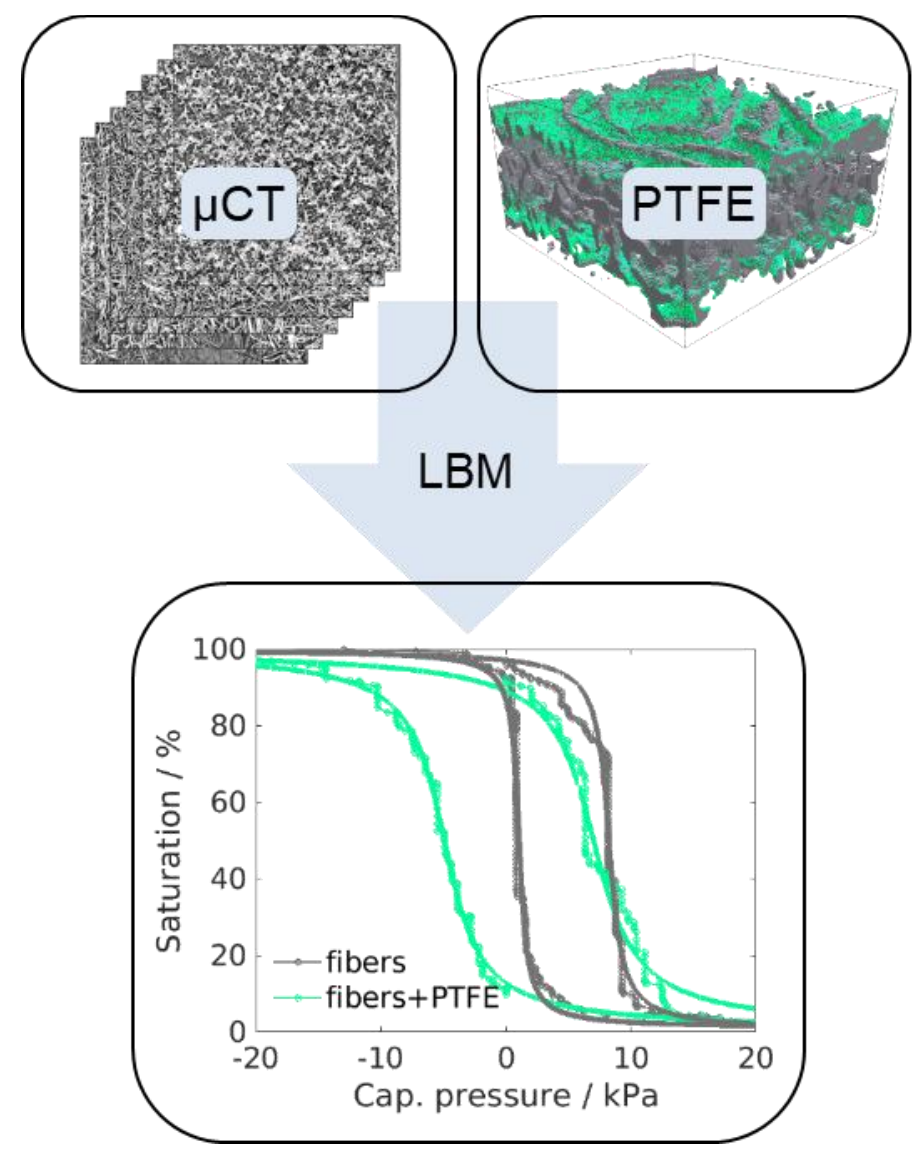

Figure 1: Workflow as developed for this research. Top left: $G D L$ microstructures are reconstructed by binarization of $\mu C T$ images. Top right: PTFE is distributed as a hydrophobic agent onto the binarized fiber structure using an in-house algorithm. 
Bottom: Effective transport parameters such as capillary pressure-saturation relations are derived by simulating liquid water transport through fibrous microstructures using the Lattice Boltzmann Method (LBM). 